\title{
Single Component, Multiphase Fluids Flow Simulation in Porous Media with Lattice Boltzmann Method
}

\author{
Xinming Zhang*
}

Department of Mathtematics and Natural Science, Harbin Institute of Technology Shenzhen Graduate School, Shenzhen, China

\begin{abstract}
In this paper, a single component, two-phase flow simulator is developed to investigate the behavior of isothermal two-phase fluid flow in porous media. The simulator is based on the lattice-Boltzmann method and the Shan-Chen multiphase model of nonideal fluids that allow coexistence of two phases of a single substance. We reproduce some different idealized situations in which the results are already known from theory or laboratory measurements and show the validity of the implementation for the physical two-phase flow in porous media. Application of the method to fluid intrusion in porous media is discussed and shows the effect of wettability on the fluid flow. The capability of reproducing critical flooding phenomena under strong wettability conditions is also proved.

Index Terms: Single Component, Multiphase Fluids, Porous Media, Simulation, Lattice Boltzmann Method

(C) 2012 Published by MECS Publisher. Selection and/or peer review under responsibility of the Research Association of Modern Education and Computer Science.
\end{abstract}

\section{Introduction}

Two-phase flows are encountered in many natural and industrial processes, including soil pollution and remediation, enhanced oil recovery, emulsion flow and stability, etc, and have considerable economic and scientific importance in these practical problems and applications. However, due to the inherent complexity of two-phase flows, from a physical as well a numerical point of view, "general" applicable computational fluid dynamics (CFD) codes are non-existent.

In the last few years the lattice Boltzmann method (LBM) which is based on the cellular automaton concept, has attracted much attention as a CFD approach in fluids engineering[1,2]. The LBM is based on statistical (macroscopic) description of microscopic phenomena. It describes a fluid as an ensemble of many particles interacting locally at the nodes of a regular lattice by collisions. And it has been shown to recover the

* Corresponding author.

E-mail address: xinmingxueshu@ gmail.com 
conservation laws of continuum fluid dynamics, and, thus, allows the calculation of the macroscopic variables such as density and velocity. For its unique derivation, LBM has some advantages over the conventional computational methods. All information transfer is local in time and space and, thus, the algorithms can easily be implemented on parallel computers. It's locality with respect to computational mesh is absolutely essential for the applications of interfacial problems. In addition, it has particular significance for porous media because LBM can easily simulate fluid flow with highly complex solid or free boundaries relative to continuum modeling approaches.

To date, a number of different approaches have been used to simulate the two-phase fluid flows, including Chromodynamic model[3], Shan-Chen model[4], free-energy model[5] and HSD model[6], etc. All these models have their own advantages in the simulation of two-phase flow. The purpose of the research presented in this paper is to investigate the behavior of isothermal two-phase fluid flow in porous media using the single component, two-phase lattice-Boltzmann model, developed by Shan and Chen. Although this model has some shortcoming, it is exceptionally versatile, and problems that have long defied quantitative treatment can now be examined. Here, we develop a two-phase flow simulator and demonstrate the applicability of the LBM for prescribing fluid properties for non-ideal fluids. We also present flow simulation results in the interior of porous media.

\section{Lattice Boltzmann Method}

Unlike traditional numerical methods which solve for the macroscopic variables, such as velocity and density, the LBM is based on the microscopic kinetic equation for the particle distribution function. The macroscopic quantities are obtained through moment integration of the distribution function. There are several different lattice-Boltzmann models available. In this paper we use the so-called lattice-BGK model[7], which is the simplest one in the hierarchy of lattice-Boltzmann methods.

The physical space is divided into a regular lattice and the velocity space is discretized into a finite set of velocities $\left\{\boldsymbol{c}_{\alpha}\right\}$, the Boltzmann equation with BGK approximation can be discretized as

$$
f_{\alpha}\left(\boldsymbol{x}_{i}+\boldsymbol{c}_{\alpha} \Delta t, t+\Delta t\right)-f_{\alpha}\left(\boldsymbol{x}_{i}, t\right)=-\frac{f_{\alpha}\left(\boldsymbol{x}_{i}, t\right)-f_{\alpha}^{e q}\left(\boldsymbol{x}_{i}, t\right)}{\tau}
$$

Where $\Delta t$ and $c_{\alpha} \Delta t$ are time and space increments, respectively. $f_{\alpha}$ is the single-particle velocity distribution function along the $\alpha$ th direction. $f_{\alpha}^{e q}$ is the equilibrium distribution function, and $\tau$ is the single relaxation time. In the simulations presented in this paper, we consider the two-dimensional square lattice with nine velocitiesD2Q9 model. In this model the equilibrium distribution function is

$$
f_{\alpha}^{e q}=\rho w_{\alpha}\left[1+\frac{3}{c^{2}} \boldsymbol{c}_{\alpha} \cdot \boldsymbol{u}+\frac{9}{2 c^{4}}\left(\boldsymbol{c}_{\alpha} \cdot \boldsymbol{u}\right)^{2}-\frac{3}{2 c^{2}} \boldsymbol{u} \cdot \boldsymbol{u}\right]
$$

where $w_{0}=4 / 9, w_{1}=w_{2}=w_{3}=w_{4}=1 / 9$, and $w_{5}=w_{6}=w_{7}=w_{8}=1 / 36$. The macroscopic density $\rho$ and velocity $\boldsymbol{u}$ are related to the distribution function by

$$
\rho=\sum_{\alpha=1}^{9} f_{\alpha} \text { and } \rho \boldsymbol{u}=\sum_{\alpha=1}^{9} f_{\alpha} \boldsymbol{c}_{\alpha}
$$

To model surface tension forces that are characterized by a non-ideal gas equation of state, a nearest and next-nearest neighbor interaction potential can be incorporated into the lattice- Boltzmann model. We have adopted the method developed by Shan and Chen, where in addition to the local collisions neighboring fluid particles exchange momentum through an attractive short-range force:

$$
\mathbf{F}(\mathbf{x}, t)=-G \boldsymbol{\psi}(\rho(\mathbf{x}, t)) \sum_{\alpha=1}^{8} w_{\alpha} \boldsymbol{\psi}\left(\rho\left(\mathbf{x}+\mathbf{c}_{\alpha} \Delta t, t\right)\right) \mathbf{c}_{\alpha}
$$

Where $\mathrm{G}$ is the interaction strength, $w_{\alpha}$ is weight coefficient, and $\psi(\rho(x, t))$ is the interaction potential: $\psi(\rho)=\psi_{0} e^{-\rho_{0} / \rho}, \psi_{0}, \rho_{0}$ are arbitrary constants. 
Adhesive forces between the fluid and solid phases are introduced into the model by Martys[8]:

$$
F_{a d s}=-G_{a d s} \psi(\mathbf{x}, t) \sum_{\alpha} w_{\alpha} s\left(\mathbf{x}+\mathbf{c}_{\alpha} \Delta t\right) \mathbf{c}_{\alpha}
$$

Here $s=0,1$ for nodes in the liquid and on solid walls, respectively. $G_{a d s}$ represents the particle interaction strength between fluid and solid walls, and varying the $G_{a d s}$ parameter allows simulation of the complete range of contact angles.

With these definitions, in simulation, the cohesive force and the attractive force are added to the velocities that compute the equilibrium distribution function with the following formula:

$$
u^{e q}=u+\Delta u=u+F_{\text {cohesive }} / \omega \rho+F_{\text {adhesive }} / \omega \rho
$$

Where $\omega=1 / \tau$ is the relaxation time, $\rho$ is the density.

\section{Results And Discussion}

The ultimate success of the LBM for simulating liquid-vapor phenomena in porous media depends on its ability to reproduce observed behavior, which for simple geometries is well described by physicochemical models. In this section, we demonstrate the applicability of the aforementioned algorithms for prescribing fluid properties in the context of the LB model for nonideal fluids. We also present the numerical simulation results of two-phase flow in porous.

Firstly, the relation between contact angle and the adhesive force constant $G_{a d s}$ was studied. Fig.1 shows the different contact angles for different adhesive force constant. As the $G_{a d s}$ decreases, we can observe that the contact angle decreases because of the adhesive force between the fluid-solid grow stronger. The simulation region is initially composed of a partial liquid droplet of radius $40 \mathrm{lu}$ on the bottom solid wall of a $200 \times 200$ lu box where the solid surface is at 1 through 2 lu in the y direction and the vapor phase elsewhere. In the simulations the no-slip boundary condition at solid-fluid interfaces is realized through a computationally efficient 'bounce-back' condition, where the particle momenta are conserved during collisions with a solid wall.

Moreover, through the simulations, we found that high value of Gads ( $G s=-100$ ) leads to compression of the liquid near surfaces causing anomalously high liquid densities. All of these simulations are together with $G=-25$ for the liquid cohesion and the particular EOS parameters.

The drainage process and imbibition process in a pipe are simulated in the Fig.2 and Fig. 3. In the simulations, the no-slip boundary condition at solid-fluid interfaces is realized through a computationally efficient 'bounce-back' condition, and periodic condition was applied in the x-direction. Fig. 2 and Fig. 3 show simple pipe models with four different diameters $(r=10,7,5,2$, respectively). We used the same values of pressure gradient ( $F=0.001)$, cohesive force and adhesive force $\left(G=-30, G_{a d s}=-110\right)$ for all models. Fig.2 shows drainage in which the non-wetting fluid is pumped into the wetting-fluid-saturated system and Fig.3 shows imbibition where the wetting fluid replaces the non-wetting fluid. As the diameter of the pipe increases, we can observe that the contact angles decrease accordingly because the capillary pressure decreases. It is also observed that the contact angle at imbibition $\left(\theta_{2}\right)$ is greater than that at drainage $\left(\theta_{1}\right)$. This is the well known contact angle hysteresis[9].The simulation successfully shows the capillary hysteresis.

The single component two-phase Lattice Boltzmann method was applied to the case of vapor displacement by liquid in a porous media. The simulations are carried out on a $230 \times 100$ lattice, and the void space of the medium is represented as a collection of large pore bodies, called chambers, interconnected through narrow capillaries called throats. Such a representation has been repeatedly proved satisfactory for a variety of 
practical materials used in many fields. The pore network is initially filled with vapor and a fluid layer is set on the first several lattices. The internal walls use a simple bounce back boundary condition. Periodic boundaries are applied in the $\mathrm{x}$ direction and quasiperiodic boundary conditions were maintained in the $\mathrm{y}$ direction.

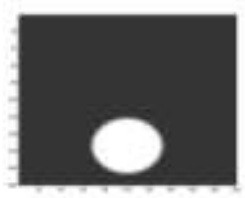

$$
\theta=180^{\circ}\left(G_{a d s}=-25\right)
$$

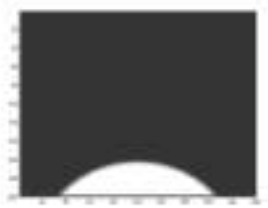

$0^{\circ}<\theta<90^{\circ}\left(G_{a d s}=-75\right)$

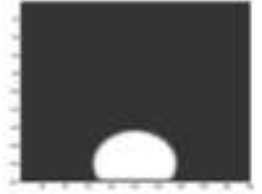

$90^{\circ}<\theta<180^{\circ}\left(G_{a d s}=-45\right)$

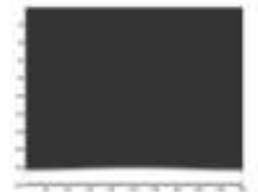

$$
\theta=0^{\circ}\left(G_{a d s}=-90\right)
$$

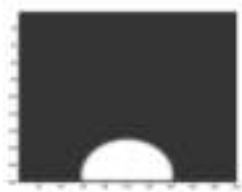

$\theta=90^{\circ}\left(G_{a d s}=-55\right)$

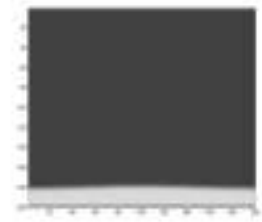

$$
\theta=0^{\circ}(G s=-100)
$$

(anomalously high liquid densities adjacent to the solid surface)

Fig. 1. Different contact angles for different adhesive force constant

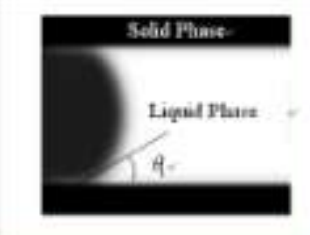

$\mathrm{R}=10$

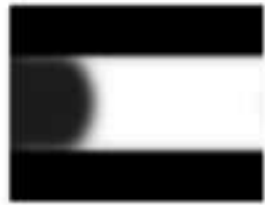

$\mathrm{R}=7$

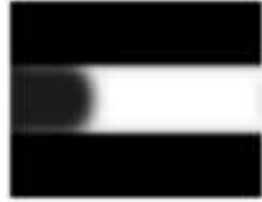

$\mathrm{R}=5$

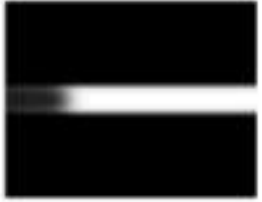

$\mathrm{R}=2$

Fig.2 Drainage process through pipes

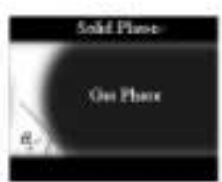

$\mathrm{R}=10$

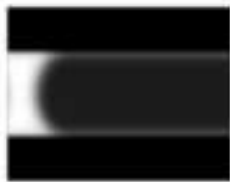

$\mathrm{R}=7$

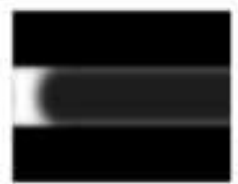

$\mathrm{R}=5$

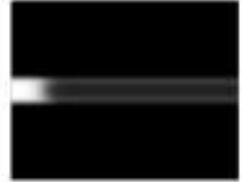

$\mathrm{R}=2$

Fig.3 Imbibition process through pipes

Two cases were studied. In the first case we consider the invasion without wettability in the system. Fig.4 shows six snapshots of the simulation results. In the second case, wettability is introduced to the system and leads to drastic changes in the displacement process. The desired wettability conditions can be implemented by assigning the surface adhesion parameter $G_{a d s}$ standing for the attractive force between fluid and solid. In this fashion, we take $G_{a d s}=-120$, namely, the contact angle $\theta=0$ (completely wet). Fig.5 shows six 
snapshots of an imbibition simulation, where the liquid phase is assumed to wet the pore surface strongly, forming a thin liquid film along the pore walls. Compared with the case 1, breakthrough is attained more easily in this experiment, especially for the narrower throat. The liquid phase advances through the pore network through, mainly, film flow resulting in poor sweeping efficiency. It seems that the sweep form can overcome the capillary resistance. The gradual increases of the film thickness causes, eventually, snap-off at pore throats and entrapment of a large vapor quantity in pore chambers[10]. Continued application of an external gravity force causes, eventually, condensation of the trapped vapor, progressively from up to down and from smaller to larger pore chambers.

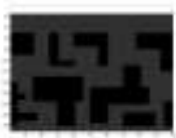

$\mathrm{T}=0$

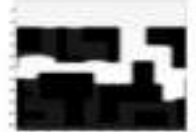

$\mathrm{T}=25000$

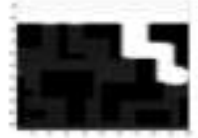

$\mathrm{T}=10000$

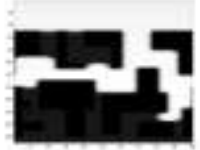

$\mathrm{T}=30000$

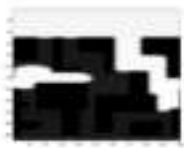

$\mathrm{T}=20000$

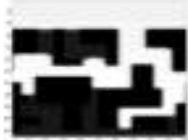

$\mathrm{T}=50000$

Fig.4 Displacement of vapor by liquid in porous media without wettability

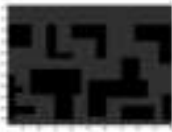

$\mathrm{T}=0$

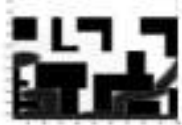

$\mathrm{T}=25000$

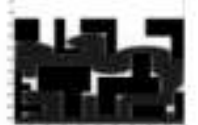

$\mathrm{T}=7000$

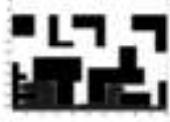

$\mathrm{T}=30000$

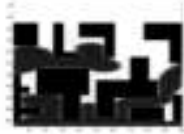

$\mathrm{T}=13000$

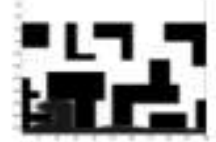

$\mathrm{T}=60000$

Fig.5 Displacement of vapor by liquid in porous media with strong wettability

\section{CONCLUSION}

To summarize, we have demonstrated the ability of single component multiphase LBM to simulate simultaneous liquid and vapor flow including contact angle and pipe flow. And the lattice-Boltzmann simulator was applied to two-phase flow problems in simple geometries and in pore networks. It was found that the simulator can predict several two-phase flow phenomena of critical significance in displacement applications, such as formation of advancing wetting film and early breakthrough under strong wettability conditions, film growth and snap off in throats, vapor condensation in pore, and wettability dependent sweeping efficiency of waterfloods. Furthermore, LBM provides insights into unstable and intermittent flows and interface routing in partially saturated pore networks that cannot be treated by standard continuum approaches such as the Richards equation. 


\section{References}

[1] Chen, S.; Chen, H.; Martinez, D. and Matthaues, W., 1991, Lattice Boltzmann model for simulation of magnetohydrodynamics, Phys. Rev. Lett., 67(27), 3776.

[2] Qian, Y. H.; d' Humières, D. and Lallemand, P., 1992, Lattice BGK models for Navier-Stokes equation, Europhys. Letters, 17(6), 479.

[3] Gunstensen, A. K.; Rothman, D. H.; Zaleski, S. and Zanetti, G., 1991, Lattice Boltzmann model of immiscible fluids, Phys. Rev. A, 43(8), 4320.

[4] Shan, X. and Chen, H., 1993, Lattice Boltzmann model for simulating flows with multiple phases and components, Phys. Rev. E, 47(3), 1815.

[5] Swift, M. R.; Osborn, W. R. and Yeomans, J. M., 1995, Lattice Boltzmann simulation of nonideal fluids, Phys. Rev. Lett., 75, 830.

[6]. He, X.; Shan, X. and Doolen, Gary D., 1998, Discrete Boltzmann equation model for nonideal gases, Phys. Rev. E, 57(1), R13

[7] Qian, Y. H.; d' Humières, D. and Lallemand, P., 1992, Lattice BGK models for Navier-Stokes equation, Europhys. Letters, 17(6), 479.

[8] Shan X. and Doolen G., 1995, Multicomponent lattice-Boltzmann model with interparticle interaction, $J$. Stat. Phys, 81, 379.

[9] Dullien, F.A.L. Porous media: Fluid Transport and Pore Structure. Academic Press, San Diego, 1992, $554 \mathrm{pp}$

[10] O. Vizika, D. G. Avraam, and A. C. Payatakes. On the role of the viscosity ratio during low-capillarynumber forced imbibition in porous media. Journal of Colloid and Interface Science. 1994, Vol.165, pp: 386401 